\title{
BURNOUT AND RISK FACTORS IN A COHORT OF NEONATOLOGISTS
}

\author{
C. Bellieni ${ }^{1}$, G. Buonocore ${ }^{1}$, C. Rosanna ${ }^{2}$, P. Righetti ${ }^{2}$, F. Iacoponi ${ }^{3}$ \\ ${ }^{1}$ University of Siena, Siena, ${ }^{2}$ Psychology, University of Padua, Padua, ${ }^{3}$ Statistics, University of Siena, Siena, \\ Italy
}

One-hundred and ten neonatologists filled in a 42-item questionnaire composed of three parts:
A)Biographic data ,
B)Personal beliefs,
C) Clinical decisions,
D) Burnout. Burnout was categorized into four subscales: psycho-physical exhaustion (PPExhaus), relationship deterioration (RelDeter), sense of professional failure (ProfFail) and Disillusion (Disill). Scores of each subscale ranged from 6 (minimum) to 36 (maximum). Burnout scales values were statistically correlated with data in points A), B), and C).

Results: Mean burnout scores are: $16.6(\mathrm{SD}=5.7), 15,3(\mathrm{SD}=4.8), 12.8(\mathrm{SD}=4.5), 12.6(\mathrm{SD}=6.7)$ for PPExhaus, RelDeter, ProfFail, and Disill respectively.

A) Biographic data: Doctors who are not parents have lower levels of RelDeter $(B=0.750 ; p=0.025)$. Doctors with less work experience have higher levels of PPExhaus $(B=-0.623 ; p=0.030)$ ProfFail $(B=-0.624$; $\mathrm{p}=0.02)$ and of RelDeter $(\mathrm{B}=0.477 ; \mathrm{p}=0.022)$.

B) Personal beliefs. Doctors who believe that living with a physical disability is unworthy have higher levels of RelDeter $(B=-0.472 ; \mathrm{p}=0.004)$. Having recurrent death ideation is more present in those who have higher levels of PPExhaus $(\mathrm{B}=1.018 ; \mathrm{p}=0.016)$, RelDeter $(\mathrm{B}=0.782 ; \mathrm{p}=0.019)$ and ProfFail $(\mathrm{B}=0.830 ; \mathrm{p}=0.029)$. Those doctors who declare to be atheist or agnostic have higher levels of ProfFail $(B=-0.792 ; p=0.012)$ and Disill $(\mathrm{B}=-0.785 ; \mathrm{p}=0.033)$ with respect to believers. A significantly higher number of doctors would resuscitate a $24 \mathrm{~W}$ rather than a $23 \mathrm{~W}(\mathrm{p}<0.001) .23 \mathrm{~W}$ resuscitation is positively influenced by working in a $3 \mathrm{rd}$ level hospital, $(\mathrm{p}=0.001)$, considering religion as important in life $(\mathrm{p}=0.028)$.

Conclusion: Burnout is at alarming levels among neonatologists; some factors we highlighted should be monitered to prevent it. 